\title{
Pre-hospital emergency medical services: a population based study of pediatric utilization
}

Anthony Suruda, Donald D Vernon, James Reading, Larry Cook, Patricia Nechodom, Daniel Leonard, J Michael Dean

\begin{abstract}
Objectives-To examine emergency medical services (EMS) usage by children in one state.

Methods-Dispatch of an EMS vehicle in response to a call in the US is referred to as a "run". Runs for Utah for 1991-92 were linked to corresponding hospital records. Abbreviated injury severity scores (AISs) were assigned using ICDMAP-90 software.

Results-For the two year period there were at least 15 EMS runs per 100 children per year, with incomplete reporting from rural areas. EMS response and scene times were similar for all age groups, but interventions were less frequent for children under 5 years of age. When the principal AIS region of injury was the head, neck, or face, cervical immobilization was less frequent for children less than 5 years of age $(54 \%)$ than for older children $(76 \%)$ and immobilization was associated with improved outcome, using the crude measure of lower hospital charges. There was a similar association between splinting of upper extremity fractures and reduced hospital charges. Both associations did not appear to be due to differences in injury severity.

Conclusions-The majority of EMS use by children is for trauma. Children less than 5 years of age are less likely to have an EMS intervention than older children. Whether the lower frequency of interventions is due to the lack of properly sized equipment on the vehicle, or to other factors, is undetermined.

(Injury Prevention 1999;5:294-297)
\end{abstract}

Keywords: emergency medical services; splints; infusions, intravenous

Intermountain Injury Control Research Center, University of Utah, Salt Lake City,

Utah

A Suruda

D D Vernon

J Reading

L Cook

P Nechodom

D Leonard

J M Dean

Correspondence to: Dr Anthony Suruda, Building 512, University of Utah, Salt Lake City, Utah 84112-0512, USA (e-mail: asuruda@dfpm.utah.edu) underserved by EMS. For example, a Kentucky study found that advanced life support interventions were performed for $25 \%$ of adolescents but only for $2 \%$ of those less than 1 year old. ${ }^{1}$ Children use ambulances less frequently than adults ${ }^{2}$ and account for approximately $10 \%$ of all EMS usage. ${ }^{34}$

The present study combined pre-hospital and hospital data for children using the EMS system in order to provide additional infor- mation concerning usage, interventions, and outcomes and to address a research need identified in the US Institute of Medicine report Emergency Medical Services for Children..$^{5}$ This question was whether data from several different components of the EMS system, such as pre-hospital care and hospital care, could be linked. If they could, this would allow additional research concerning pre-hospital care, such as analysis of intervention for specific anatomic sites of injury.

\section{Methods}

DATA FILES

When an ambulance is dispatched in response to a call for assistance in the US, this is commonly referred to as an "EMS run". EMS run reports for Utah for 1991-92 were obtained from the Bureau of EMS, Utah Department of Health. All EMS records were complete except for those from several rural counties, comprising $8 \%$ of the population. Reports for canceled runs were excluded. Hospital records were obtained and included demographic information, length of stay, primary and additional diagnoses, and charges. Diagnostic and charge information was complete for all children for 1992; for 1991, this information was complete only for children with trauma.

\section{PROBABILISTIC RECORD LINKAGE}

The analysis database was created by probabilistically linking the EMS, discharge, and emergency department data using the linkage described by Jaro. ${ }^{6}$ By comparing several common fields using an iterative approach, databases are linked mathematically. The variables used for linkage in this study were first and last name, date of incident, date of birth, hospital code, county code, gender, and age. Multiple linkage passes were done using differing groups of these variables as blocking variables. Successful linkage is related to whether a person was actually injured, errors in the data sources, and the effectiveness of the algorithm used. Because the EMS database includes persons not transported to a hospital, a substantial portion of the EMS database cannot be linked with an inpatient or emergency department record.

\section{DATA ANALYSIS}

Diagnostic categories were assigned from the hospital inpatient or outpatient primary diagnoses to which the run was matched, rather than from the EMS diagnosis. Rates of use for children were calculated using 1991 and 1992 census projection data for Utah. Abbreviated 
Table 1 Mean EMS times by age (all runs in $\mathrm{min}$ )

\begin{tabular}{lclll}
\hline Age group & Response time & Scene time & Transport time & Total time \\
\hline $0-4$ & $10.2(7.9)$ & $20.1(19.0)$ & $16.9(12.0)$ & $38.4(24.6)$ \\
$5-9$ & $8.4(6.5)$ & $16.2(12.0)$ & $14.2(10.6)$ & $31.8(15.4)$ \\
$10-14$ & $8.8(7.0)$ & $16.3(9.2)$ & $13.8(11.3)$ & $31.4(15.7)$ \\
$15-17$ & $8.7(7.0)$ & $16.3(9.2)$ & $12.6(11.1)$ & $31.5(16.1)$ \\
\hline
\end{tabular}

Table 2 EMS procedures per run (\%) (excludes interfacility transfers)

\begin{tabular}{lllll}
\hline Age group & IV access & $\begin{array}{l}\text { Airway } \\
\text { management }\end{array}$ & Given medication & $\begin{array}{l}\text { Mean No of EMS } \\
\text { procedures }\end{array}$ \\
\hline $0-4$ & 8 & 3.5 & 10 & 1.54 \\
$5-9$ & 10 & 2.1 & 10 & 2.11 \\
$10-14$ & 13 & 1.8 & 14 & 2.24 \\
$15-17$ & 16 & 1.9 & 17 & 2.31 \\
All children & 12 & 2.5 & 13 & 2.01 \\
\hline
\end{tabular}

IV $=$ intravenous.

Table 3 Pre-hospital interventions for children with trauma by severity of injury

\begin{tabular}{llll}
\hline ISS (\% of runs) & $\begin{array}{l}\text { Proportion having } \\
I V^{\star} \text { procedure (\%) }\end{array}$ & $\begin{array}{l}\text { Proportion with } \\
\text { airway } \\
\text { management (\%) }\end{array}$ & $\begin{array}{l}\text { Proportion with } \\
\text { ALSt care (\%) }\end{array}$ \\
\hline $1(62)$ & 6 & 1 & 21 \\
$2-9(32)$ & 19 & 2 & 37 \\
$10-24(4)$ & 52 & 8 & 69 \\
$25-75(2)$ & 58 & 43 & 77 \\
\hline
\end{tabular}

${ }^{\star} \mathrm{IV}=$ intravenous; $\nmid \mathrm{ALS}=$ advanced life support.

injury severity scores (AISs) and injury severity scores (ISSs) were assigned from hospital discharge diagnoses using ICDMAP-90 software developed by MacKenzie et $a l^{7}$ and Tri-Analytics Inc.

Analyses of hospital charges, various EMS times, and length of hospital stay were done after excluding the upper $1 \%$ of observations to eliminate excessively high values that might unduly influence the mean. Analysis of ISSs was done after eliminating patients with missing ISS values and any with ISS $=0$, which indicates that a valid ISS could not be assigned by the software.

In urban areas of Utah it is common for more than one EMS vehicle to be dispatched upon receipt of a call and for the child to be transported by either a basic life support (emergency medical technician) or an advanced life support (or paramedic) provider depending upon the severity of injury or illness. Determination of whether advanced life support level care was provided for a particular call was made from the coding on the EMS run form.

\section{Results}

During the two year period, there were 20272 EMS runs for 15724 children, an average of 1.3 vehicle responses per child, and a use rate of 15 runs per 1000 children per year. The linkage rate of EMS to hospital records was $80.1 \%$. Approximately $7 \%$ of runs for children were to destinations other than hospitals, and another $4 \%$ of runs were canceled, so $11 \%$ of runs would not be expected to be linked.

Overall, $38 \%$ of runs provided advanced life support level service. There was a higher likelihood of this level of service when several EMS responders were identified (62\%) than when a hospital record linked to only a single responder $(32 \%$, odds ratio $(\mathrm{OR})=$ $3.46,95 \%$ confidence interval (CI) 3.18 to 3.76). This is consistent with Utah's tiered response system.

The majority of EMS transports were for trauma $(76 \%)$ rather than illness $(24 \%)$. The proportion attributable to trauma would have been only $49 \%$ if the EMS diagnosis was used rather than the hospital diagnosis. The hospital admission rate for all children brought by EMS was $26 \%$, and was higher for children less than 5 years of age $(32 \%)$ and those transported by advanced life support providers (38\%).

EMS response times and scene times were greater for children less than 5 years of age than for older children $(p<0.05$, table 1$)$. Increased scene time was not due to an increased number of EMS procedures, such as intravenous treatment. Some of this increase is explained by the greater number of interfacility transfers for younger children. When interfacility transfers via EMS were excluded from the analysis, the scene times were the same. The data did not include information on attempted but unsuccessful procedures.

Pre-hospital EMS interventions and level of care are shown in table 2. Except for airway management, the interventions were less frequent for children less than 5 years of age. EMS interventions for children with trauma were more frequent when injury severity was greater, as shown in table 3. There was an increase of 3.5 minutes in average scene time when intravenous treatment was started, and an increase of 4.5 minutes when airway management was used.

For children in whom head, neck, or face was the principal body region of maximum AIS, and who were transported by EMS from a scene rather than from a health care facility, cervical immobilization was done in $62 \%$ of cases, a spine board was applied in $56 \%$ of cases, and $68 \%$ were immobilized by either or both means. Cervical immobilization was more likely to be done for children in this group who were older than 5 years of age than for younger children (OR $=2.6,95 \%$ CI 1.9 to 3.4$)$. It was not possible to determine from the electronic EMS run report whether an appropriate size cervical collar or other immobilization device for young children was available on the EMS vehicle, or whether an appropriate size was used.

For children in whom head, neck, or face was the principal body region with the maximum AIS, mean total hospital charges were greater in those who did not have cervical immobilization by EMS ( $\$ 2304 v \$ 1898$ ), again after excluding interfacility transfers. Children who had cervical immobilization by EMS had slightly higher mean ISSs (3.6 v 3.4). The association between not having cervical immobilization and higher hospital charges remained when children less than 5 years of age, who were less likely to have cervical immobilization, were excluded from the analysis, and also when the analysis was restricted to children with ISS scores from 1-9 only. 
Table 4 Injury severity, hospital charges, and length of stay for children whose principal body region of injury was the upper extremity

\begin{tabular}{llcl}
\hline & Mean ISS & $\begin{array}{l}\text { Mean hospital } \\
\text { charge (\$) }\end{array}$ & $\begin{array}{l}\text { Mean length of } \\
\text { stay (days) }\end{array}$ \\
\hline Arm injury with splinting & 3.3 & 695 & 1.4 \\
Arm injury without splinting & 2.6 & 1195 & 2.2 \\
Arm fracture with splinting & 4.3 & 984 & 1.4 \\
Arm fracture without splinting & 4.6 & 2218 & 2.5 \\
\hline
\end{tabular}

For children whose principal body region of injury by AIS was the upper extremity, splinting was performed in $28 \%$ of cases. For those whose principal body region of injury was the lower extremity, splinting was performed in $31 \%$ of cases. Splinting was less frequent in those under 5 years of age $(\mathrm{p}<0.05)$.

When the upper extremity was the principal region of injury, splinting was associated with significantly lower hospital charges and length of hospital stay $(\mathrm{p}<0.05)$, and this was not explained by severity of injury (table 4 ). The majority of fractures involved the forearm. When children less than 5 years of age, for whom splinting was applied less frequently, were excluded from the analysis, the relationship of splinting to charges and length of hospital stay was not materially changed.

Mortality of children transported by EMS during the study period during the acute period and six month follow up was less than $1 \%$. There were too few deaths for detailed analysis.

\section{Discussion}

In this study we have shown the value of linking EMS and hospital records to define the epidemiology of EMS use by children. Using the hospital International Classification of Diseases, 9th revision, diagnoses for children transported by EMS allows identification of specific medical conditions, injuries by anatomic location, and estimation of injury severity. This provides important descriptive information and can be the basis for examining outcomes related to interventions. Determination of diagnosis from hospital rather than EMS diagnosis is consistent with the methodology used in the Child and Adolescent Emergency Department Visit Databook, ${ }^{8}$ and is in agreement with other studies. $^{9}$

We have shown that children less than 5 years of age receive fewer pre-hospital EMS interventions. Whether this is due to lack of proper sized equipment, the limited ability of EMS personnel to properly assess emergencies in very young children, or to other factors, is not known.

That $10 \%$ of EMS runs were interfacility transfers is comparable with the $12 \%$ reported during transport to a level I pediatric trauma center. ${ }^{10}$

We found variation in EMS scene times by age group that was not explained by performing EMS procedures but which was related to age. Addition of coding to the EMS run form that would identify attempted procedures that were unsuccessful would assist in analyzing the increased scene times, noting that certain unsuccessful attempts at procedures, such as attempting intravenous procedures, could be expected to take longer than a successful attempt, particularly in young children.

It appears that appropriate level care was rendered to more severely injured children, as provision of advanced life support care was related to increasing injury severity as judged by ISS, which is consistent with previous reports. ${ }^{11}$

EMS interventions for trauma patients increased with severity of injury (table 3 ). Whether interventions such as establishment of intravenous access by EMS personnel contribute to improved outcome for injured children is unknown and has been reported to be inconsequential. ${ }^{12}$

Analysis of specific EMS interventions suggested that outcome was improved by immobilization, if hospital charges and length of hospital stay are considered indicators of outcome (table 4). There were too few observations for specific fractures and other anatomic injuries for a more detailed comparison of children who were immobilized and those who were not.

Whether this association is causal, or whether it is related to other factors, is unknown. It is possible that certain injuries that are less severe are also easier to immobilize. ISS scoring may not accurately gauge minor extremity injuries. Provision of coding for nontraditional immobilization on EMS run forms would allow identification of immobilization of extremities by buddy splinting to the leg, or by taping an arm to an intravenous board. These are standard interventions for young children for which EMS treatment codes were not used during the study period.

The frequency of interventions for young children is consistent with a 1991 review of pediatric EMS systems that reported that in most communities, EMS personnel did not receive the initial training needed to care for children. ${ }^{13}$

The descriptive findings of this study are limited by the retrospective use of existing data, missing EMS data for approximately $8 \%$, the omission of most canceled EMS runs that did not result in patient transport, and the use of automated AIS and ISS scoring. ISS scoring has been criticized as not being "child friendly" because scores are derived from adult norms. ${ }^{14}$ The ICDMAP90 software used for this study included age in the calculation of AIS and ISS. This new version may be more useful for pediatric trauma research than previous methods.

We did not have available measures of long term outcome such as impairment or functional limitation, or measures of satisfaction with care. Hospital charges and length of hospital stay are related to both injury severity and outcome. That we could examine the association of EMS procedures to hospital outcomes is an indication of the usefulness of data linkage for improving EMS outcomes research. 
The authors wish to thank the Utah Hospital Association, each of the individual Utah hospitals, and the Bureau of EMS of the Utah Department of Health for sharing data. We also wish thank Jan Buttrey, Lisa Carlson, Breck Rushton, and Gerald Van Orman of the Bureau of EMS for continuing consultation and advice with this project.

This study was funded by the Agency for Health Care Policy and Research award number ROI HS09057.

1 Svenson JE, Nypaver M, Calhoon R. Pediatric prehospital care. Pediatr Emerg Care 1996;12:173-9.

2 Maio RF, Tedeschi P, Swor R, et al. Regional variation of nonrural pediatric ambulance transport rates: an ecological study. Pediatr Emerg Care 1996;12:277-82.

3 Seidel JS, Henderson DP, Ward P, et al. Pediatric prehospital care in urban and rural areas. Pediatrics 1991;88:68190.

4 Tsai A, Kallsen G. Epidemiology of pediatric prehospital care. Ann Emerg Med 1987;16:284-92.

5 Institute of Medicine. Emergency medical services for children. Washington: National Academy Press, 1993.

6 Jaro M. Probabilistic linkage of large public health data files. Stat Med 1995;14:491-8.
7 MacKenzie EJ, Steinwachs DM, Shankar B. Classifying trauma severity based on hospital discharge diagnossifying trauma severity based $1989 ; 27: 412-9$.

8 Weiss HB, Mathers LJ, Forjuoh SN, et al. Child and adolescent emergency department visit databook. Pittsburgh: Center for Violence and Injury Control, Allegheny University of the Health Sciences, 1997.

9 Schafermeyer RW. Prehospital curriculum development: a learning objective approach. Pediatr Emerg Care 1993;9:40-5.

10 Brady W, Hennes H, Wolf A, et al. Pattern of basic life support ambulance use in an urban pediatric population. $A m \mathcal{F}$ Emerg Med 1996;14:250-3.

11 Foltin GL, Pon S, Tunik M, et al. Pediatric ambulance utilization in a large American city: a systems analysis approach. Pediatr Emerg Care 1998;14:254-8.

12 Teach SJ, Antosia RE, Lund DP, et al. Prehospital fluid therapy in pediatric trauma patients. Pediatr Emerg Care 1995;11:5-8.

13 Foltin G, Fuchs S. Advances in pediatric emergency medical systems. Emerg Med Clin North Am 1991;9:459-74.

14 Beattie TF, Currie CE, Williams JM, et al. Measures of injury severity in childhood: a critical overview. Inj Prev 1998;4:228-31.

\section{Light fantastic}

Without the colour photograph, the impact of this piece is lessened but just use your imagination!

A 10 year old boy was admitted to the hospital with crampy epigastric pain after eating. He mentioned swallowing a small object. Gastroscopy revealed a toy flashlight $(2.5 \mathrm{~cm}$ in diameter) emblazoned with a cartoon character. The boy's symptoms were relieved after the flashlight was removed, and his flashlight still worked.

The photo shows a grinning gremlin-like character shining up from within body organs (letters to the editor, New England fournal of Medicine, 29 July, 1999: 378).

Housework as dangerous male occupation

To illustrate the dangers of ironing in front of the telly ... A gentleman in Dubbo was engaged in this task when the phone rang. He answered the iron rather than phone and managed to steam press his ear (Australian Magazine, 28-29 August, 1999: 56).

\section{A day in the life of the Royal Ulster Constabulary}

- 264 "999" emergency calls

- 210 crimes recorded

- 57 crimes cleared

- 41 domestic incidents attended

- £19000 worth of drugs seized

- Nine parades or demonstrations policed

- Five attacks carried out against police officers

- 327 fixed penalty notices issued to motorists

- 20 road traffic accidents reported

- 90000 miles travelled by police vehicles

(Extracted from RUC annual report 1998-99)

(Contributed by regional correspondent Rosie Mercer, who comments, "If only the road safety campaigners could get their hands on a small portion of the fines collected from the fixed penalty system (a rough and probably underestimate of 327 tickets $\times 365$ days @ £20 each = $£ 2387$ 100), we would be able to support greater levels of research and action to combat some of the problems.) 